\title{
Analysis of Offshore Knuckle Boom Crane - Part Two: Motion Control
}

\author{
Morten K. Bak Michael R. Hansen
}

Department of Engineering Sciences, Faculty of Engineering and Science, University of Agder, 4879 Grimstad, Norway. E-mail: \{morten.k.bak,michael.r.hansen\} @uia.no

\begin{abstract}
In this paper design of electro-hydraulic motion control systems for offshore knuckle boom cranes is discussed. The influence of the control valve bandwidth along with the ramp time for the control signal are investigated both analytically with simplified system models and numerically with an experimentally verified crane model.

The results of both types of investigations are related to general design rules for selection of control valves and ramp times and the relevance of these design rules is discussed. Generally, they are useful but may be too conservative for offshore knuckle boom cranes. However, as demonstrated in the paper, the only proper way to determine this is to evaluate the motion control system design by means of simulation.
\end{abstract}

Keywords: Hydraulic crane, system design, directional control valve, bandwidth, ramp time

\section{Introduction}

Design of offshore knuckle boom cranes is a complex and multidisciplinary task involving mechanical, hydraulic and control systems design. Naturally, this is an iterative process as the design of the crane's mechanical system and motion control system depend on each other. In practice though, detailed design of both systems is carried out separately as concurrent activities with constraints imposed by a conceptual design. This paper focuses on design of the motion control system.

Despite the fact that hydraulics, in general, is considered a mature technology, design of hydraulic motion control systems still offers a number of challenges for both component suppliers and manufacturers of hydraulically actuated machines. For the system designer, the main challenge is to meet the functional requirements for the system, a set of design constraints, while satisfying a number of performance criteria such as cost, reliability, overall efficiency and controllability, which are often conflicting and also subject to con- straints. Design and optimization of hydraulic system has been subjected to quite extensive research, e.g., by Krus et al. (1991), Andersson (2001), Hansen and Andersen (2001), Krimbacher et al. (2001), Stecki and Garbacik (2002), Papadopoulos and Davliakos (2004), Pedersen (2004) and Bak and Hansen (2013b).

Besides design of the actuation system, there are generally three elements to take into account when designing electro-hydraulic motion control systems:

- Control strategy.

- Control elements (control valves).

- Reference signals (generation and shaping).

In this paper a typical control strategy for offshore knuckle boom cranes is considered and the selection of control elements and reference signals are investigated in order to identify their influence on the system performance. 


\section{Motion Control System Design}

Offshore knuckle boom cranes generally feature a high degree of automation compared to other types of cranes. The control strategy relies on position and/or velocity feedback from the individual degrees of freedom (DOFs). For DOFs actuated by hydraulic cylinders this is usually achieved by means of a position sensor integrated in the cylinder. Fig. 1 shows the general architecture of a typical electro-hydraulic motion control sub-system for the considered type of crane.

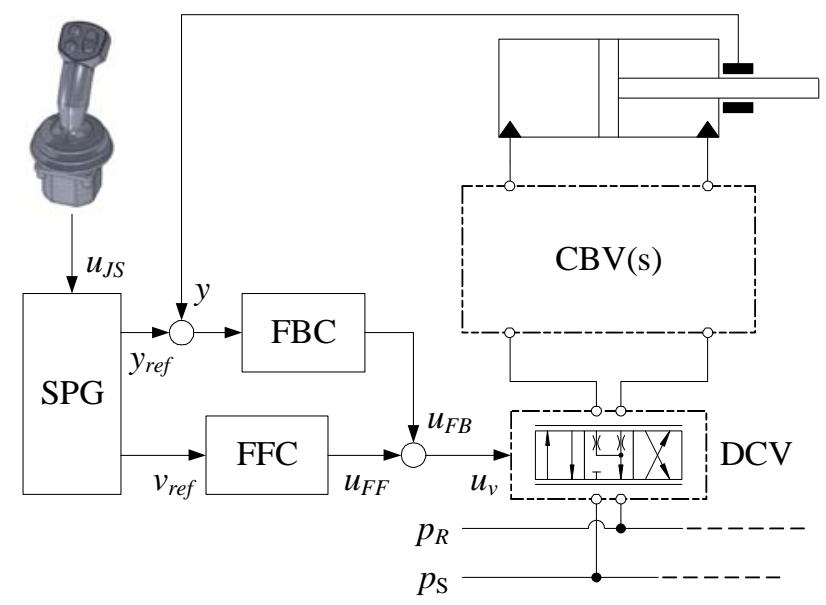

Figure 1: Simplified schematic of electro-hydraulic motion control sub-system.

The cylinder motion is controlled with a $4 / 3$ directional control valve (DCV) which controls the flow into either of the two cylinder chambers. However when the cylinder is exposed to negative loads (piston velocity and load force have the same direction), also the outlet pressure of the cylinder needs to be controlled. For that a counterbalance valve $(\mathrm{CBV})$ is used which provides a relief valve functionality on the outlet side assisted by the pressure on the inlet side. If negative loads can occur in both directions of motion, CBVs are required on both the piston side and the rod side of the cylinder. CBVs exist in different variations, e.g., externally vented, non-vented and relief compensated, due to the different applications they are used for. In Bak and Hansen (2013a) these valve are discussed in further detail.

The DCV may either be a servo valve or a proportional valve with closed loop spool position control and either with or without pressure compensation. Most often a pressure compensated DCV is used, like the one for the intermediate and outer jibs of the considered crane (see Bak and Hansen (2013a)). The reason for this is that it provides load independent flow control which simplifies the control system and makes it easier to tune the controller gains. Furthermore, load independent flow control is required whenever an operator is to control more than one DOF at the time without assistance from the control system.

The control system consists of four elements:

- Human-machine interface (HMI).

- Set point generator (SPG).

- Feedforward controller (FFC).

- Feedback controller (FBC).

Besides monitors, push buttons and switches the HMI contains two joysticks which the operator uses to generate command signals for the control system. Joystick signals are fed to the SPG where they may be treated in different ways depending on the selected control mode. In open loop control mode the joystick signal, $u_{J S}$, is fed directly to the DCV as a feedforward signal. In closed loop control mode joystick signals are transformed into velocity and position references for the individual cylinder motions. The latter is used for path control of the crane's gripping yoke where several DOFs are controlled in a coordinated manner.

The FFC is a scaling of the velocity reference with the feedforward signal, $u_{F F}$, given by:

$$
u_{F F}=v_{r e f} \cdot K_{F F}
$$

The feedforward gain, $K_{F F}$, is a tuning parameter, but may be computed when using a pressure compensated DCV. With a DCV with linear flow characteristics the cylinder velocity is given by:

$$
v_{c y l} \approx \frac{u_{v} \cdot Q_{\max }}{A_{Q}}
$$

$Q_{\text {max }}$ is the maximum flow of the DCV and $A_{Q}$ is either the piston area or the rod-side area of the cylinder, depending on the sign of the valve control signal, $u_{v}$, i.e., the sign of the velocity reference, $v_{r e f}$ :

$$
A_{Q}=\left\{\begin{array}{lll}
A_{p} & \text { for } & 0 \leq u_{v} \\
A_{p} \cdot \phi & \text { for } & u_{v}<0
\end{array}\right.
$$

The cylinder area ratio is $\phi=\left(D_{p}^{2}-D_{r}^{2}\right) / D_{p}^{2}$, where $D_{p}$ is the piston diameter and $D_{r}$ is the rod diameter. Combining (1) and (2) yields the feedforward gain:

$$
K_{F F}=\frac{A_{Q}}{Q_{\max }}
$$

Theoretically, the cylinder could be controlled by the feedforward controller alone. However due to internal leakages in the hydraulic system, deadband of the DCV and nonlinear characteristics of the main spool this is 
not possible in practice.

The FBC is a PI controller which compensates for disturbances and accumulated position errors. The control system usually also contains an element which compensates for deadband of the DCV. This deadband compensator, however, is not considered here.

The system architecture shown in Fig. 1 is a popular structure because of its simple and, consequently, robust design. Furthermore, the controllers are easy to tune because of the load independent flow control.

\section{Dynamic Considerations}

A critical point in the design process is the selection of the DCV and, more specifically, to determine which dynamic properties are required from the valve. According to Merritt (1967) the bandwidth of the DCV should be greater than any of the natural frequencies of the hydraulic-mechanical system it is used to control. Manufacturers of servo valves usually recommend choosing a valve with a bandwidth, $\omega_{v}$, which is at least three times higher than the natural frequency, $\omega_{h m}$, of the hydraulic-mechanical system (MOOG, 2012):

$$
\omega_{v} \geq 3 \cdot \omega_{h m}
$$

This applies if the valve bandwidth should not affect the overall bandwidth of the total system consisting of the valve and the hydraulic-mechanical system it is used to control. Furthermore, it applies for servo applications where fast response and high precision is required and where non-compensated DCVs are used. In order to investigate the relevance and usefulness of the design rule stated by (5) a simplified model of a hydraulic servo system is considered. The system structure is illustrated by the block diagram in Fig 2 which corresponds to a single DOF of the considered crane.

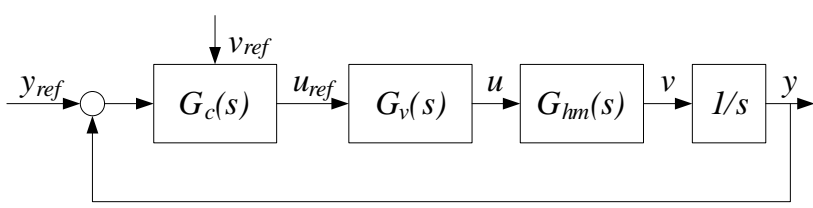

Figure 2: Block diagram representation of hydraulic servo system controlling a single DOF.

The $G_{c}$ block represents the controller and contains both the FFC and the FBC. The $G_{v}$ block represents the DCV including the closed loop spool position control and the dynamics of the valve. For simplified analysis this block is normally modeled as a second order system:

$$
G_{v}(s)=\frac{1}{\frac{s^{2}}{\omega_{v}^{2}}+\frac{2 \cdot \zeta_{v} \cdot s}{\omega_{v}}+1}
$$

The $G_{h m}$ block is the hydraulic-mechanical system to be controlled, in this case also represented by a second order system:

$$
G_{h m}(s)=\frac{K_{h m}}{\frac{s^{2}}{\omega_{h m}^{2}}+\frac{2 \cdot \zeta_{h m} \cdot s}{\omega_{h m}}+1}
$$

The performance of any hydraulic servo system depends on the bandwidth of these two series connected systems, i.e., the product of the two transfer functions in (6) and (7). If the design rule in (5) is followed the overall dynamics of the valve and the hydraulicmechanical system will approximately be that of the hydraulic-mechanical system. If the valve has a smaller bandwidth then the overall bandwidth of the system is markedly reduced. In Fig. 3 the Bode plot of $G_{h m}$ is shown together with the product $G_{v} \cdot G_{h m}$ for $\omega_{v}=3 \cdot \omega_{h m}$ and $\omega_{v}=\omega_{h m}$. The remaining system parameters are $K_{h m}=1, \omega_{h m}=10 \mathrm{rad} / \mathrm{s}, \zeta_{h m}=0.2$ and $\zeta_{v}=0.8$

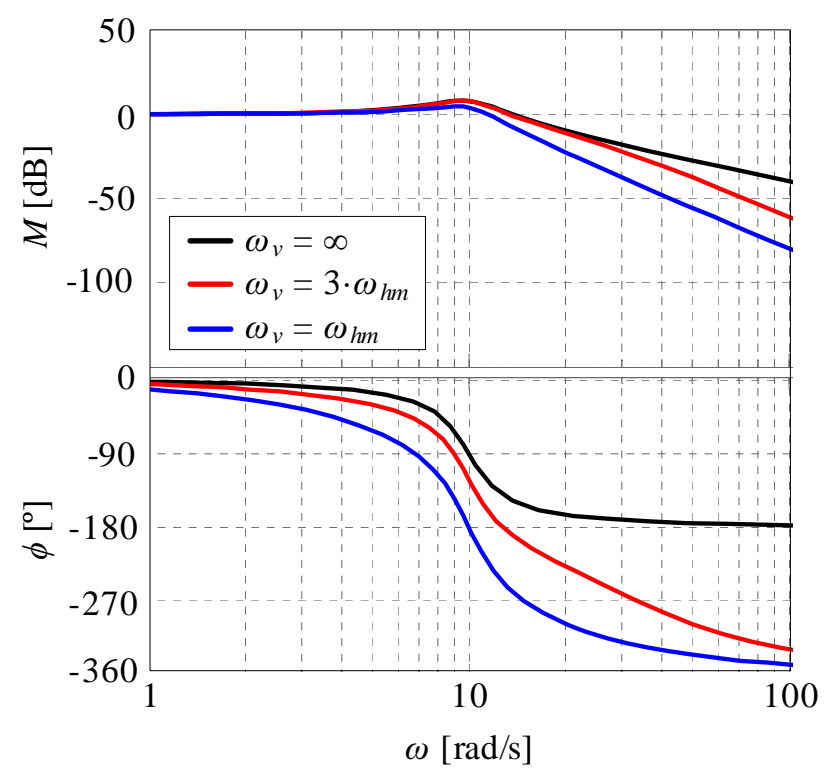

Figure 3: Bode plot of different combinations of valve dynamics and hydraulic-mechanical system dynamics.

Clearly, choosing $\omega_{v}=\omega_{h m}$ yields a system with distinctly poorer dynamic performance whereas choosing $\omega_{v}=3 \cdot \omega_{h m}$ justifies the simplification:

$$
G_{v h m}(s)=\frac{K_{h m}}{\frac{s^{2}}{\omega_{v h m}^{2}}+\frac{2 \cdot \zeta_{h m} \cdot s}{\omega_{v h m}}+1} \approx G_{v} \cdot G_{h m}
$$


where

$$
\omega_{v h m}=0.9 \cdot \omega_{h m}
$$

Equation (8) and (9) are valid for frequencies up to and around $\omega_{h m}$. Frequencies beyond this value are rarely of interest for hydraulic servo systems. Therefore, when applying (5), the valve dynamics can simply be disregarded and the effective bandwidth (natural frequency) of the total system, $\omega_{v h m}$, may be used as a design reference, which is $90 \%$ of $\omega_{h m}$.

Besides the bandwidth of the DCV also the motion reference for the control system must be considered. Also here the natural frequency of the hydraulicmechanical system must be taken into account as it gives an indication of the limit that the system imposes on the desired motion.

Consider the mass-spring-damper system shown in Fig. 4 with a mass, $m$, a stiffness, $k$, and a damping, $b$. This may be represented by an underdamped second order system with a natural frequency, $\omega_{n}$, and a damping ratio, $\zeta$. The mass is traveling at a speed, $\dot{y}=v_{0}$, and the motion of the mass should be ramped down via the reference input, $x$. In principle, this corresponds to halting a hydraulically controlled payload by ramping down the input flow.

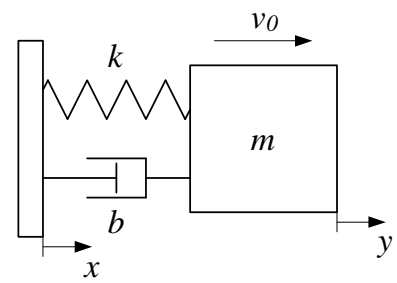

Figure 4: Second order system with a mass travelling at a speed $\dot{y}=v_{0}$.

Initially, $\dot{x}=\dot{y}=v_{0}$ and then the reference velocity is ramped down according to:

$$
\dot{x}=v_{0} \cdot\left[1-\frac{t}{t_{r}}\right], \quad t \leq t_{r}
$$

Here $t$ is the time and $t_{r}$ is the ramp time during which the mass is decelerated. The analytical solution to the motion of the mass during the ramp down is:

$$
\begin{aligned}
y & =C_{0}+C_{1} \cdot t+C_{2} \cdot t^{2} \\
& -C_{0} \cdot e^{-\alpha \cdot t} \cdot\left[\cos (\beta \cdot t)+\frac{\alpha}{\beta} \cdot \sin (\beta \cdot t)\right]
\end{aligned}
$$

where

$$
C_{0}=\frac{v_{0}}{\omega_{n}^{2} \cdot t_{r}} \quad C_{1}=v_{0} \quad C_{2}=-\frac{v_{0}}{2 \cdot t_{r}}
$$

and

$$
\alpha=\zeta \cdot \omega_{n} \quad \beta=\omega_{n} \cdot \sqrt{1-\zeta^{2}}
$$

The reference velocity in (10) becomes zero at $t=t_{r}$. At that instant the position error, referred to as the overshoot, is:

$$
\begin{aligned}
e & =\left|x_{t=t_{r}}-y_{t=t_{r}}\right| \\
& =\frac{v_{0}}{\omega_{n}^{2} \cdot t_{r}} \cdot\left\{1-e^{-\alpha \cdot t_{r}} \cdot\left[\cos \left(\beta \cdot t_{r}\right)+\frac{\alpha}{\beta} \cdot \sin \left(\beta \cdot t_{r}\right)\right]\right\}
\end{aligned}
$$

The relative overshoot is the absolute position error relative to the nominal travel:

$$
\begin{aligned}
e_{r e l} & =\frac{e}{x_{t=t_{r}}} \\
& =\frac{2}{\omega_{n}^{2} \cdot t_{r}^{2}} \cdot\left\{1-e^{-\alpha \cdot t_{r}} \cdot\left[\cos \left(\beta \cdot t_{r}\right)+\frac{\alpha}{\beta} \cdot \sin \left(\beta \cdot t_{r}\right)\right]\right\}
\end{aligned}
$$

In Fig. 9 the relative overshoot is plotted as a function of the ramp time for two different damping ratios. The natural frequency has no influence on the curves.

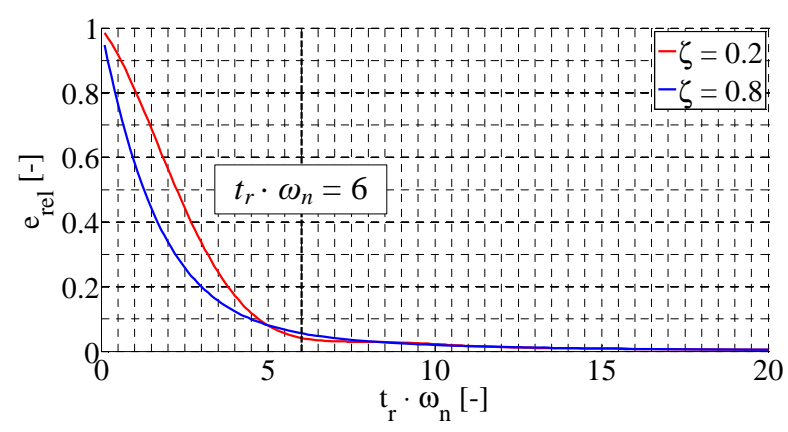

Figure 5: Relative overshoot as function of ramp time for two different damping ratios.

Most hydraulic-mechanical systems have dynamic characteristics that are not significantly more complicated than for the system in Fig. 4. Therefore the result shown in Fig. 5 may be utilized for hydraulic servo systems. It is clear that for a typical hydraulicmechanical system, ramp times should be considered whenever motion is prescribed. Also, it is clear that a useful ramp time depends on the acceptable overshoot. Hence, if high overshoot is acceptable then small ramp times may be prescribed and vice versa. As a rule of thumb, the ramp time of a prescribed motion should obey the inequality:

$$
t_{r} \geq \frac{6}{\omega_{n}}
$$

It is important to keep in mind that the natural frequency of a real system may be substantially smaller than the one computed for a model of the system. The discrepancy is mainly because the modeled stiffness typically is higher than the actual stiffness. This 
can be taken into account in many ways, for example by using a smaller stiffness in the computation or by means of experimental work that can reveal the actual natural frequency of the system to be controlled.

\section{Practical Application}

With the experimentally verified simulation model described in Bak and Hansen (2013a) it is possible to generate a realistic picture of the natural frequency of the crane. Theoretically, there may be several mode shapes and natural frequencies for the crane. In practice, though, it will only be possible to excite the lowest of these frequencies. This frequency will be common for all the considered DOFs, i.e., the ones for three crane jibs, but will vary with positions of the jibs, i.e., the lengths of the hydraulic cylinders.

By orienting the crane jibs in different positions in the simulation model and applying an impulse the natural frequency for the given positions can be observed from the following pressure oscillations in the cylinders. As seen from Fig. 6 the crane is a redundant mechanism, since there are more DOFs than needed to position the gripping yoke within the work space of the crane. This redundancy is not exploited for control purposes but only to improve foldability. Therefore, the cylinders 2 and 3 are simply operated equally in practical operations.

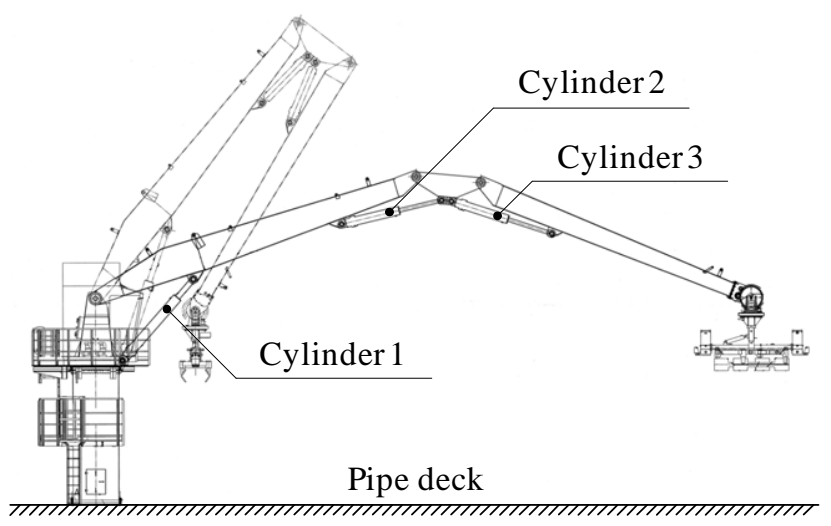

Figure 6: Knuckle boom crane with redundant DOF.

The variation of the natural frequency can be mapped in a three-dimensional representation as shown in Fig. 7 as function of only two independent cylinder lengths.

The figure provides a clear picture of how the natural frequency varies with the length of the cylinders. The orientation of the inner jib has no major influence, except when cylinders 2 and 3 are nearly fully extended. The natural frequency varies between $f_{h m} \approx 0.65 \ldots 1.9$ $\mathrm{Hz}$ corresponding to $\omega_{h m} \approx 4 \ldots 12 \mathrm{rad} / \mathrm{s}$.

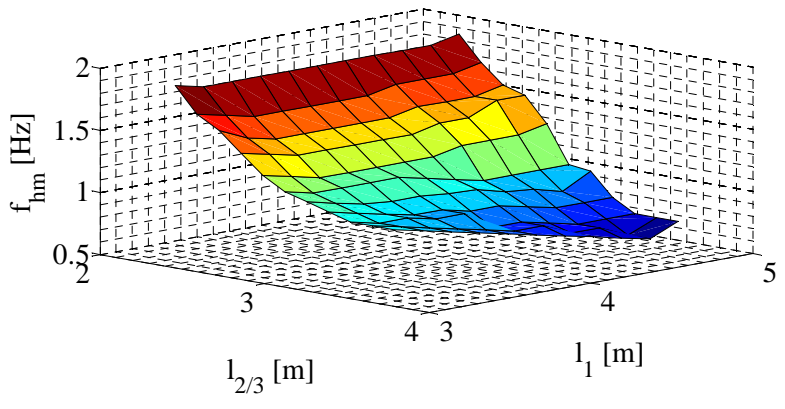

Figure 7: Variation of natural frequency of the crane.

In order to investigate how well the simplified analysis in section 3 applies to a real system like the considered crane, simulations with parameter variations are carried out with the experimentally verified crane model. To limit the extent of the analysis a simple sequence of extending the outer jib cylinder is considered. While cylinders 1 and 2 are held at rest cylinder 3 is extended with trapezoidal velocity reference, see Fig. 8, using the FFC only. The FBC is disabled to ease comparison and the deadband of DCV is set to zero in order to simulate the function of a deadband compensator.

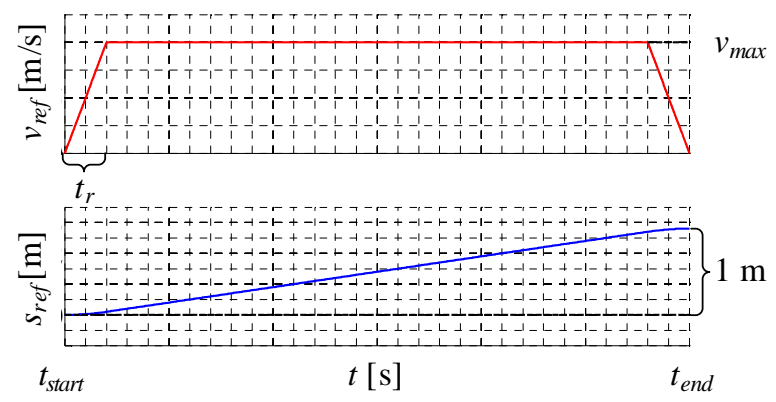

Figure 8: Velocity and position references.

The reference cylinder travel is $1 \mathrm{~m}$, starting and ending $0.15 \mathrm{~m}$ from the end stops. In this range the natural frequency varies between $f_{h m} \approx 1.65 \ldots 0.65 \mathrm{~Hz}$ corresponding to $\omega_{h m} \approx 10 \ldots 4 \mathrm{rad} / \mathrm{s}$. Since the highest natural frequency is present at the beginning of the sequence only the performance during the ramp up is considered and $\omega_{h m}=10 \mathrm{rad} / \mathrm{s}$ is used as the reference frequency. According to (5) the DCV bandwidth should then be at least $\omega_{v}=30 \mathrm{rad} / \mathrm{s}$, which is at the performance limit of most pressure compensated DCVs. Bak and Hansen (2012) tested a Danfoss PVG32 and identified a bandwidth of $f_{v}=5 \mathrm{~Hz}$ or slightly more than $30 \mathrm{rad} / \mathrm{s}$.

Simulations are carried out with variations of both 
ramp time, $t_{r}$, and $\mathrm{DCV}$ bandwidth, $\omega_{v}$, where relative position error at the end of the ramping period and maximum position error during the sequence are observed. In Fig. 9 the relative position error is shown as function of $t_{r} \cdot \omega_{h m}$ for different DCV bandwidths.

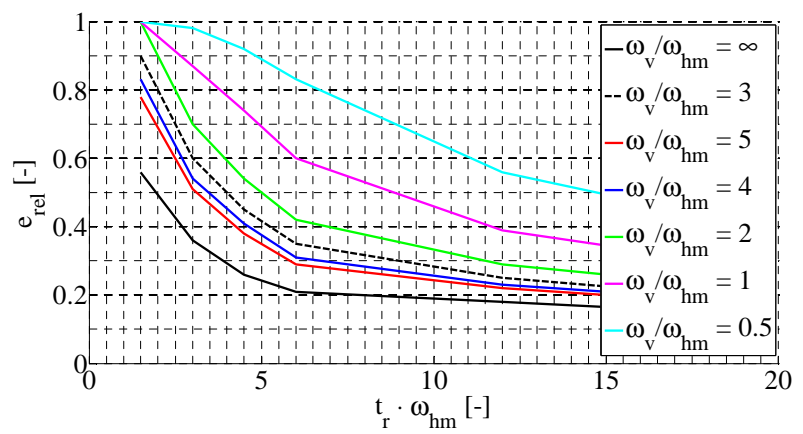

Figure 9: Relative error at the end of the ramp.

The effect is the similar to the one seen in Fig. 5. The relative error increases significantly for $t_{r} \cdot \omega_{h m}<6$ at least for $\omega_{v}>\omega_{h m}$. Furthermore, it is seen that $e_{r e l}$ is doubled by choosing $\omega_{v}=2 \cdot \omega_{h m}$ and tripled for $\omega_{v}=\omega_{h m}$ compared to the ideal situation of $\omega_{v}=\infty$. Only minor improvements are achieved for $\omega_{v}>3 \cdot \omega_{h m}$. The results seem to support the design rules in (5) and (16).

In Fig. 10 the maximum position error is shown as function of $t_{r} \cdot \omega_{h m}$ for different DCV bandwidths.

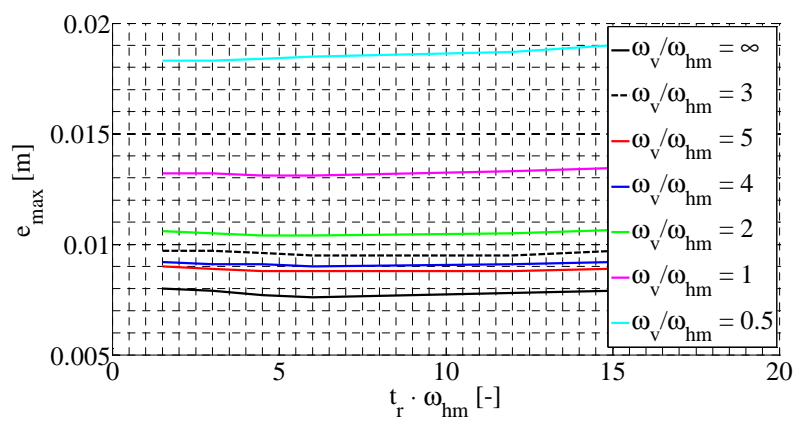

Figure 10: Maximum error during the sequence.

The maximum position error is almost independent of the ramp time and only depends on the DCV bandwidth. This indicates that if the relative error is irrelevant, then the ramp time does not need to be taken into account. However too short ramp times may cause instability while too long ramp times increase the maximum reference velocity and consequently the required flow.

The results once again support the design rules in (5) and (16) since there is no significant improvement for $\omega_{v}>3 \cdot \omega_{h m}$, but a significant increase in error for $\omega_{v}=\omega_{h m}$ compared to $\omega_{v}=3 \cdot \omega_{h m}$.

The absolute errors are small compared to the reference travel of the cylinder and well within the acceptable range for typical offshore knuckle boom crane operations. The simulation results confirm the validity of (5) and (16) and usefulness as general design rules. In general, the choice of DCV and ramp times will always depend on the acceptable levels of relative and absolute errors. A prerequisite to evaluate this is to have a simulation model, like the one described in Bak and Hansen (2013a), with which a frequency map, like the one in Fig. 7, can be generated and relevant control sequences can be simulated.

\section{Conclusions}

In this paper design of electro-hydraulic motion control systems for offshore knuckle boom cranes has been discussed and a typical system architecture has been presented. A critical point in the design process is the selection of the directional control valve (DCV) and more specifically to determine which dynamic properties are required from the valve. A commonly used design rule is to select a valve with a bandwidth, $\omega_{v}$, which is at least three times higher than the natural frequency, $\omega_{h m}$, of the hydraulic-mechanical system it is used to control.

The influence of the DCV bandwidth along with the ramp time for the control signal have been investigated both analytically with simplified system models and numerically with the experimentally verified crane model described in Bak and Hansen (2013a).

The results of both types of investigations confirm the relevance and usefulness of design rules for required DCV bandwidth and suitable ramp times. However the selection of these design parameters always depend on the acceptable error level for the application to be controlled and for offshore knucke boom crane the investigated design rules may be too conservative.

However, the only way to properly evaluate the performance of the motion control system design, without building a prototype, is to have simulation model of the application like the one described in Bak and Hansen (2013a).

\section{Acknowledgements}

The work presented in this paper is funded by the Norwegian Ministry of Education and Research and Aker Solutions. 


\section{References}

Andersson, J. Multiobjective optimization in engineering design. Applications to fluid power systems. Ph.D. thesis, Linköping University, Linköping, Sweden, 2001.

Bak, M. K. and Hansen, M. R. Modeling, performance testing and parameter identification of pressure compensated proportional directional control valves. In Proceedings of the 7th FPNI PhD Symposium on Fluid Power. Reggio Emilia, Italy, pages 889-908, 2012.

Bak, M. K. and Hansen, M. R. Analysis of offshore knuckle boom crane - part one: modeling and parameter identification. Modeling, Identification and Control, 2013a. 34(4):157-174. doi:10.4173/mic.2013.4.1.

Bak, M. K. and Hansen, M. R. Model based design optimization of operational reliability in offshore boom cranes. International Journal of Fluid Power, 2013b. $14(3): 53-66$.

Hansen, M. R. and Andersen, T. O. A design procedure for actuator control system using design optimization. In Proceedings of the 7th Scandinavian International Conference on Fluid Power. Linköping, Sweden, 2001.
Krimbacher, N., Garstenauer, M., and Scheidl, R. Optimization of hydraulic systems by means of numerical simulation. In Proceedings of the 2nd International Workshop on Computer Software for Design. Ostrava-Malenovice, Czech Republic, pages 76-83, 2001.

Krus, P., Jansson, A., and Palmberg, J.-O. Optimization for component selection in hydraulic systems. In Proceedings of the 4 th Bath International Fluid Power Workshop. Bath, UK, 1991.

Merritt, H. E. Hydraulic control systems. Wiley, 1967.

MOOG. Electrohydraulic valves... a technical look. Technical report, Moog Inc., 2012. URL http://www.moog.com/literature/ICD/ Valves-Introduction.pdf.

Papadopoulos, E. and Davliakos, I. A systematic methodology for optimal component selection of electrohydralic servosystems. International Journal of Fluid Power, 2004. 5(3):15-24.

Pedersen, H. C. Power management in mobile hydraulic applications - an approach for designing hydraulic power supply systems. In Proceedings of the 3rd FPNI PhD Symposium on Fluid Power. Terrassa, Spain, pages 125-139, 2004.

Stecki, J. S. and Garbacik, A. Fluid Power Net Publications, 2002. 